research article

\title{
Increased late urinary toxicity with whole pelvic radiotherapy after prostatectomy
}

\author{
Borut Kragelj \\ Department of Radiotherapy, Institute of Oncology Ljubljana, Ljubljana, Slovenia
}

\begin{abstract}
Background. Radiotherapy aimed at prostatic bed (PBRT) can prevent recurrence or reestablish remission in prostate cancer patients primarily treated with prostatectomy. In selected patients results may be improved with the additional irradiation of pelvic nodes (WPRT).

Patients and methods. The objective of the study was to evaluate late toxicity of postoperative radiotherapy in 43 patients - 21/43 treated with WPRT. Dysuria, haematuria, nocturia, continence and obstructive urination problems as well as urgency, continence, frequency, pain and bleeding of defecations were prospectively registered and converted to a modified Radiation Therapy Oncology Group (RTOG) - late effects normal tissue (LENT) scoring system. Median tumour dose (TD) for PBRT was 64.8 (59.4-70.0) Gy and for WPRT 50.4 (48.0-56.0) Gy.

Results. More important than the deterioration of intestinal function (worsening for 1 grade in $54 \%$ and $\geq 2$ grades in $5 \%$ of patients) was the deterioration of urinary function (worsening for 1 grade in $33 \%$ and $\geq 2$ grades in $26 \%$ of patients). This appeared to be more frequent in patients with WPRT than PBRT (67\% vs. $50 \%$ of patients) especially in conjunction with WPRT TD $>52$ Gy (deterioration in $71 \%$ of patients). Conclusions. Although several factors may influence increased urinary toxicity after WPRT, it seems reasonable to lower the urinary bladder dose as it possible with novel radiation techniques.
\end{abstract}

Key words: prostate cancer; postoperative radiotherapy; whole pelvic radiotherapy; late toxicity

\section{Introduction}

Radiotherapy has a well established role in the treatment of patients after radical prostatectomy. Immediately after the operation it can prevent recurrence in patients with high risk features ${ }^{1-3}$ or reestablish the

Received 15 March 2009

Accepted 31 March 2009

Correspondence to: Borut Kragelj, MD. PhD, Department of Radiotherapy, Institute of Oncology Ljubljana, Zaloška c. 2, SI-1000 Ljubljana, Slovenia; Phone: +386 15879 489; Fax: +386 15879 400; E-mail: bkragelj@onko-i.si remission when applied as a salvage treatment for biochemical or local recurrence. ${ }^{4}$ It can be used as a sole treatment or in combination with hormonal therapy. ${ }^{5}$

Regardless whether it is used immediately postoperatively, or as a salvage treatment, radiation fields are focused on a believable position of prostate and seminal vesicles as it was before the resection ${ }^{1-3,6,7}$ and/or areas of the most probable local recurrence. ${ }^{8}$

It is suggested that with broadening of the treatment fields with the inclusion of pelvic nodes in selected patients considered at high risk of lymph node involvement, the 
improved biochemical relapse-free survival can be acquired. ${ }^{9}$

The objective of the study was to evaluate late toxicity of this treatment.

\section{Patients and methods}

Documents of 49 patients with prostate cancer that received the radiation treatment after prostatectomy between June 2001 and December 2005 at Institute of Oncology Ljubljana were analysed to evaluate the consequences of the radiation treatment. The treatment and the follow-up with the attentive evaluation of side effects were conducted by the author.

Radiation started 1.8-88.5 (median 6.1) months after prostatectomy. Forty-three $\%(21 / 49)$ of patients were treated a few months after prostatectomy because of a high risk of recurrence and with preradiation PSA below $0.2 \mathrm{ng} / \mathrm{ml}$; the others $(28 / 49$ (57\%) patients) were submitted to radiotherapy because of the biochemical or local recurrence.

Treatment fields were limited to prostatic bed (PBRT) in 24/49 (49\%) of patients - in 25/49 (51\%) patients pelvic nodes up to the lumbosacral (S1/L5) interspace were also included (WPRT). The median dose defined by $95 \%$ isodose, encompassing target volume applied to the prostatic bed, was 64.8 Gy (59.0-70.0 Gy) and 50.4 Gy (48.0$56.0 \mathrm{~Gy})$ to the pelvic region in fractions of 1.8-2.2 Gy (median 2.0 Gy). Bioequivalent doses for a fraction of 2 Gy $\left(\mathrm{BED}_{2}\right)$ were computed using $\alpha / \beta=3$. Median $\mathrm{BED}_{2}$ to the prostatic bed was 62.2 Gy (range 57.373.0 Gy) and to the whole pelvis $50 \mathrm{~Gy}$ (dose range 48.0-57.3 Gy). Treatments were delivered on the linear accelerator using four-field technique and standard fractionation. A dose calculation with 2D planning was performed in 20/49 (41\%) patients and $3 \mathrm{D}$ in $29 / 49$ (59\%) patients. The posterior rectal wall was excluded on lateral fields otherwise no dose constraints were used to limit the dose to organs at risk. Regular portal images were used to limit set up errors.

Long term side effects were assessed in $43 / 49$ (88\%) patients still alive and with no sign of recurrence at the time of evaluation.

Side effects rising as a consequence of urinary damage were prospectively registered at each visit with regard to dysuria, haematuria, nocturia, incontinence and obstructive problems. Similarly, the consequences of intestinal toxicity were registered with regard to urgency, continence, frequency of defecations, as well as pain and bleeding problems.

Side effects were converted to late lower gastrointestinal and urinary toxicity scores according to Storey's modification of the Radiation Therapy Oncology Group (RTOG) - late effects normal tissue (LENT) late toxicity scoring system at the time of evaluation. ${ }^{10}$ Still some additional modification was performed regarding urinary frequency and obstruction primarily aimed to evaluate toxicity already present after prostatectomy. A minor modification was made also with regard to intestinal toxicity with the inclusion of urgency of defecations (Table 1). Erectile problems were not prospectively evaluated and therefore, they were not included and analysed.

Urination characteristics of the initial (before radiotherapy) and the final (at the last follow-up visit) evaluation were compared also in the sense of the possible improvement. On the other hand, worsening of urination characteristics for a grade was considered as a minor deterioration, while worsening of two or more grades was considered as a major deterioration. The same principle was applied also in the evaluation of late intestinal toxicity.

The Kaplan Meier survival method was used to assess the time of the appearance of toxicity. The first appearance of the most 
Table 1. Modifications of delayed radiation toxicity grading using Radiation Therapy Oncology Group (RTOG) and Late Effects Normal Tissue Task Force (LENT) criteria with regard to urinary and intestinal toxicity

\begin{tabular}{lllll}
\hline Criteria & Grade1 & Grade 2 & Grade 3 & Grade 4 \\
\hline $\begin{array}{l}\text { Nocturnal } \\
\text { urinary } \\
\text { frequency }\end{array}$ & $2-3$ & $3-4$ & hourly or less & $\begin{array}{l}\text { dysfunction requiring } \\
\text { cystectomy/urinary } \\
\text { diversion/nephrostomy }\end{array}$ \\
$\begin{array}{l}\text { Obstructive } \\
\text { urinary } \\
\text { symptoms }\end{array}$ & occasional & regular & $\begin{array}{l}\text { operative } \\
\text { treatment / } \\
\text { urethrotomy }\end{array}$ & $\begin{array}{l}\text { dysfunction requiring } \\
\text { cystectomy/urinary } \\
\text { diversion/nephrostomy }\end{array}$ \\
$\begin{array}{l}\text { Urinary } \\
\text { incontinence }\end{array}$ & occasional/drops & $\begin{array}{l}\text { regular us of up to } \\
1 \text { sanitary pad }\end{array}$ & $\begin{array}{l}\text { regular use of } 2 \\
\text { sanitary pads or } \\
\text { more }\end{array}$ & $\begin{array}{l}\text { dysfunction requiring } \\
\text { cystectomy/urinary } \\
\text { diversion/nephrostomy }\end{array}$ \\
$\begin{array}{l}\text { Urgency of } \\
\text { defecations }\end{array}$ & $\begin{array}{l}\text { present/ without } \\
\text { incontinence }\end{array}$ & $\begin{array}{l}\text { intermittent use of } \\
\text { sanitary pads }\end{array}$ & $\begin{array}{l}\text { regular use of } \\
\text { sanitary pads }\end{array}$ & $\begin{array}{l}\text { dysfunction requiring } \\
\text { surgery }\end{array}$ \\
\hline
\end{tabular}

pronounced change in urination and defecation characteristics in each patient was considered as the observed event. 1-cummulative survival curve was used to present the results graphically. Chi square test was used for the estimation of differences in late toxicity between patients with prostate only and additional pelvic radiation as well as between patients with different dose regimens. ${ }^{11}$ P-value $\leq 0.05$ was considered significant in all statistical tests. The SPSS 15.0 for Windows was used as a tool for the analysis.

\section{Results}

Considerable urination difficulties already existed at the start of the radiation treatment. These were mostly related to the increased nocturnal urination frequency and difficulties with continence. Problems with increased nocturnal urinations experienced
21/43 (49\%) patients - difficulties were considerable (grade 3 ) in $5 / 43(12 \%)$. Even more pronounced were efforts to remain continent. More or less pronounced dripping of urine was evident in 24/43 (56\%) patients. Urinary incontinence without the need for sanitary pads was evident in 14/43 (33\%) patients, up to one sanitary pad per day was used by the next 6/43 (14\%) patients while pronounced incontinence with 2 or more pads per day was evidenced in $4 / 43(9 \%)$ patients.

Late toxicity was evaluated after a median follow up of 52 months. During follow up visits some of urination difficulties have actually resolved or became less pronounced (Table 2). The improvement in the sense of less frequent nocturnal urinations was noted in 9/21 (43\%) patients with this problem after the surgery and the improvement in the sense of less pronounced incontinence was noted in 11/24 (46\%) pa-

Table 2. Change in urination characteristics after postoperative radiotherapy comparing the condition after prostatectomy and at the last follow-up

\begin{tabular}{lccccc}
\hline $\begin{array}{l}\text { Change in urination } \\
\text { characteristics }\end{array}$ & Dysuria & Haematuria & Nocturia & Incontinence & Obstruction \\
\hline Improvement & 0 & 0 & 9 & 11 & 0 \\
No change & 40 & 37 & 18 & 20 & 40 \\
Minor deterioration & 2 & 3 & 12 & 7 & 1 \\
Major deterioration & 1 & 3 & 4 & 5 & 2 \\
\hline
\end{tabular}


tients who had continence difficulties after the surgery.

The comparison of all urination characteristics between the situation at the start of radiation and at the last follow up visit showed that some improvement, or no change, was evident in 18/43 (42\%) patients, and minor deterioration was noticed in $14 / 43(33 \%)$ patients. However, the major deterioration of urination characteristics was evident in 11/43 (26\%) patients. These eleven patients included two patients with severe haemorrhagic cystis and 6 patients with G3 urinary toxicity. In both patients with haemorrhagic cystitis cystectomy was obligatory. However, in 2 of 6 patients with G3 toxicity symptoms have resolved after the endoscopic incision for the bladder neck obstruction.

As expected, in comparison to crude incidence rates, results of the survival analysis of toxicity events were even more unpromising showing the deterioration of urinary function in $73 \%$ of patients at 5 years (Figure 1).

The comparison of the incidence of deteriorations between patients that received PBRT and WPRT, as well as the comparison of $\mathrm{BED}_{2}$ for WPRT (lower or greater than $52 \mathrm{~Gy}$ ) and of $\mathrm{BED}_{2}$ for prostate bed (lower or greater than $65 \mathrm{~Gy}$ ) showed no statistical significance. However, differences were seen in favour of PBRT and also in favour of lower radiation doses to either prostate bed or pelvic nodes (Table 3).

Compared to urinary deterioration, the deterioration of the intestinal function was

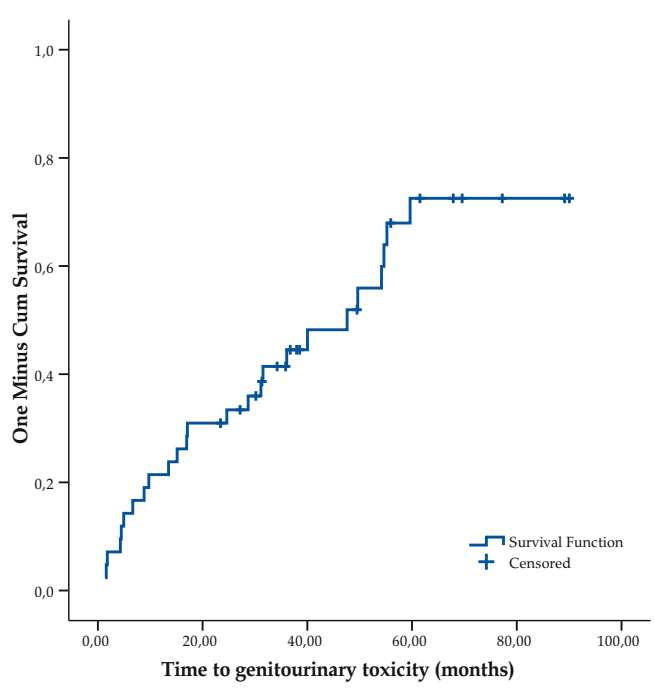

Figure 1. Survival without deterioration of urination characteristics.

markedly less pronounced. It appeared as more frequent defecations (in 23/43 (54\%) patients), urgency to defecate usually as a consequence to some dietary offence (in 15/43 (35\%), occasional bleeding (7/43 (16\%) or occasional uncontrolled mucous discharge (in 4/43 (9\%) patients). None of the patients experienced G3/G4 toxicity. Altogether defecation characteristics remained unchanged in 13/43 (30\%) patients, the minor deterioration as G1 toxicity was evident in 28/43 (65\%) and major as G2 toxicity in $2 / 43(5 \%)$ patients.

Actuarial rates of intestinal radiation late effects were slightly lower than for the urinary tract $(63 \%$ at 5 years) and with decreasing appearance of new cases after 40 months after the start of radiotherapy.

Table 3. Incidence of deterioration in urinary function regarding treatment fields and radiation doses

\begin{tabular}{lll}
\hline Characteristics & Incidence of deteriorations & P \\
\hline Prostate bed RT & $11 / 22(50 \%)$ & 0.226 \\
Whole pelvic RT & $14 / 21(67 \%)$ & \\
Whole pelvic dose $<52$ Gy & $8 / 13(62 \%)$ & 0.243 \\
Whole pelvic dose 252 Gy & $6 / 8(75 \%)$ & \\
Prostate bed dose $<64$ Gy & $15 / 29(52 \%)$ & 0.259 \\
Prostate bed dose 264 Gy & $10 / 14(71 \%)$ & \\
\hline
\end{tabular}


No difference in the rate of side effects was found between pelvic and prostatic only radiation and also not between different dosages

\section{Discussion}

Postoperative radiotherapy is generally reported as non toxic with a few severe late side effects. With treatment fields limited to prostatic bed and doses of 60-62 Gy, rates of serious side effects are lower than $5 \%, 2,12$ and seems to remain within this range also with the dose escalation to 70 Gy. ${ }^{13,14}$

However, low grade toxicity seems to be quite common. In the survey of 75 patients, Pearce reported the incidence of any toxicity registered at any point during the median follow up of 45 months, after the irradiation of 60-66 Gy to prostatic bed, to be $51 \%$ for intestinal and $78 \%$ for urinary tract. ${ }^{15}$ Similarly in the report of EORTC trial 22911 5-year cumulative incidence rate of complications (of any grade) based on the competive risk analysis in the radiotherapy arm is about $70 \% .^{2}$ Doses applied in the trial were 60 Gy while treatment fields were limited more or less to prostatic areas. Initial 50 Gy were applied to somewhat larger area including the region of seminal vesicles and larger margins around prostatic bed. Compared to these reports our results are disappointing. They do not differ so much in overall toxicity as in the rate of severe complications. A straightforward explanation for these events is hard to give. Especially if we consider that both patients that required cystectomy were treated with conventional doses of 60 and 64 Gy and fractionation of $2 \mathrm{~Gy}$ and with treatment fields limited to the initial position of prostate and seminal vesicles.

Long term intestinal toxicity, although common in our study, does not turn out to be an important problem. In all patients it was of a low grade. In the majority it was manifested with slightly increased number of defecations. They also needed some kind of diet, and in the case of an offence, urgency that may in some patients be aggravated to minor continence problems occurred. No difference was noted whether treatment fields were limited to a prostate region or they encompass also pelvic nodes.

Prevalent problems in our patients were related to postirradiation damage to urinary bladder. However, our results suggest that considerable problems with urination were already present after the prostatectomy. Furthermore, results of already mentioned EORTC trial suggest that problems arise with time also when prostatectomy was the sole treatment. So, we can not look at the consequences of the postoperative radiation neglecting the impact of the surgical treatment. At least in a part, long term side effects of this combined treatment are due to the prostatectomy. The improvement of urination characteristics in $24 \%$ of patients after radiotherapy suggests a prolonged healing after the prostatectomy at least in some patients. For these patients often used interval of 2-3 months to start adjuvant radiation after the surgery may be too short. According to this, lower toxicity could be expected in patients irradiated after an interval that would suggest a complete healing after the prostatectomy. However, there was no difference in the rates of major deterioration of urination characteristics between patients irradiated with an interval to surgery shorter or longer than 2 years. It appeared in 5/15 (33\%) and 6/28 (21\%) patients respectively. Nevertheless, an uncompleted healing after the surgery may be an additional factor contributing to long term toxicity. It seems reasonable to wait with irradiation until patients report no further improvement, perhaps jointly with the introduction of the hormonal treatment. 
Further reasons for urination problems may ground in the properties of the postoperative irradiation. Limited data are available as a predictor of late urinary toxicity in the postoperative radiotherapy. ${ }^{12}$ Less obscured is a situation with the radical radiotherapy treatment of prostate cancer and some of these data may be valid also in the context of postoperative radiotherapy. Pinkawa stated in a study of 80 patients with the use of Expanded Prostate Cancer Index Composite questionnaire, that the patients' ability to fill the bladder has a major impact on the dose-volume histogram parameters and on both, acute and late urinary toxicity. ${ }^{16}$ Similar was the conclusion of Harsolia stating the importance of urinary bladder dose-volume parameters on 331 patients using National Cancer Institute Common Toxicity Criteria 2.0 for the evaluation of chronic urinary toxiciy. ${ }^{17} \mathrm{~A}$ further similarity of both studies is a finding of the importance of the exposure of urinary bladder to relatively low doses of radiation in the range of 30-40 Gy. In our study, due to the inclusion of pelvic nodes and the use of box technique, large parts of bladder were exposed to irradiation. Even when treatment fields were restricted to prostatic bed regularly about $80 \%$ of the bladder volume received dose equal or greater than $60 \mathrm{~Gy}$ (dose that is similar to target doses). The interdependence of late urinary toxicity and volume and dose of irradiation is also suggested by our results. The increase in urinary problems, although not statistically significant, was seen in patients with larger fields by the comparison of pelvic and prostate only irradiation. The increase in toxicity was also evident with larger doses - greater than 52 Gy to pelvic field and 64 Gy to prostatic bed. To reduce the radiation damage to urinary bladder it seems important not only to limit the target dose but also, and for most, to exclude as much as possible of the bladder out of the irradiated volume and to limit the dose to the rest of the bladder wall. New radiation techniques such as intensity modulated radiotherapy (IMRT) can produce concave dose distribution and, as shown by planning studies, can reduce the volume of the bladder exposed to high doses for $20-50 \% .{ }^{18,19}$ Less effective is IMRT in the low dose range. How effective will be this and other techniques that make it possible to reduce the dose to the urinary bladder, in the reduction of late urination toxicity with pelvic irradiation is still unclear.

Perhaps the easiest and most reliable way to solve the marked urinary toxicity is to reduce the target volume. EORTC giudelines ${ }^{8}$ for the target volume definition in postoperative radiotherapy for prostate cancer, with the limitation of target volume to the sites of most probable local recurrence, enable a considerable reduction in dose-volume parameters of the urinary bladder. However, certain assurance is needed to exclude pelvic nodes. Reliable information is offered by extended, and perhaps also, but with less trustworthiness, classic lymphadenectomy. ${ }^{20}$ In our study lymphadenctomy was performed in $77 \%$ of patients with a median 6 resected nodes. If, and how much, the omitment of pelvic radiotherapy in our patients would compromise the results of the treatment, could be anticipated from the initial results that suggest the significantly improved progression free survival (86\% and $71 \%$ at 5 years) in patients treated with whole pelvic radiotherapy (WPRT) for the relapsed or progressive disease after the prostatectomy. They support the effectiveness and superiority of WPRT to prostatic bed radiation for patients with the risk of nodal invasion greater than 15\% according to Roach equation ${ }^{21}$ and with no reliable information on the lymph node status through lymphadenectomy. More secure is to make some compromise in the target volume. One possibility is the less strict 
inclusion of the lymphatic region around a distal part of external iliacal vessels, ${ }^{22}$ and the other is to adapt target volume to the extensiveness of lymphadenectomy with the exclusions of regions that were already submitted to lymphadenectomy.

The hormonal therapy can be a component of postoperative treatment. ${ }^{23}$ In the majority of our patients, goserelin $10.8 \mathrm{mg}$ every 3 months was used for one year. The treatment started at least one month before the commencement of radiotherapy, so castrate testosterone levels were achieved at the time of radiotherapy. Considering the study of Taussky in which a relationship between chronic toxicity and testosterone level at the time of radiotherapy was stated, hormonal therapy should also be considered as one of the possible factors that influence our results. ${ }^{24}$ What will be the risks of the exclusion of the immediate hormonal therapy will be eventually demonstrated by RTOG 96-01 trial results.

Nevertheless, some caution is needed with the interpretation of the results on late urinary toxicity. The fibrosis of the bladder wall and the loss of muscle function as a result of late radiation damage, were in the majority of our patients demonstrated with continence problems and increased urination frequency with the need to wake up at night. Common criteria used to evaluate and grade this problem, are toxic criteria of the Radiation Therapy Oncology Group (RTOG) and the European Organization for Research and Treatment of Cancer. ${ }^{25}$ However, in RTOG scales these problems are almost completely ignored, as is the case with incontinence, or inexact (and subjective) for correct grading of the problem, such is the case with the increased urination frequency. Modifications of this scoring system as proposed by Storey ${ }^{10}$ also leave some uncertainties in the evaluation of mild, initial continence problems not necessitating the use of pads, and also severe problems with the use of multiple pads per day. It also remains too broad to register changes in the urination frequency - especially in connection with the postoperative radiation of prostatic carcinoma with distinctive problems present already after the prostatectomy. Not so rare scenario of 2-3 wakening per night after the prostatectomy with the increase to $4-5$ wakening can be scored as no toxicity (not twice baseline), G1 toxicity (twice baseline) and also as G2 or G3 (moderate or severe) toxicity depending on the impression of the inquirer. One can speculate that exaggerated urinary toxicity in our study can be the consequence of more rigorous grading - even with the regard to, generally more exact, grading of serious toxicity.

As a conclusion we can say that pelvic radiotherapy in the postoperative treatment of prostatic carcinoma may result in the increased and perhaps unacceptable urinary morbidity at least in connection with a classic box technique of irradiation and concurrent testosterone deprivation. Like in other irradiated areas, toxicity is strongly correlated with the irradiated volume in conjunction with the dose. ${ }^{26}$ The quality of life became more and more important in the case of a good prognosis of patients' outcomes regarding a disease control and survival, therefore, we have to carefully look for late toxicity assessing restrictions in the combined modality treatment. ${ }^{27}$ However, there are several possibilities to make pelvic radiotherapy after the radical prostatectomy more acceptable. Nevertheless, the individual response to radiotherapy is important, even with regard to high grade morbidity.

\section{Acknowledgement}

The author thanks Dr. Lijana ZaletelKragelj from Department of Public Health, Ljubljana University Faculty of Medicine, 
Slovenia, for her help in preparation of this manuscript.

\section{References}

1. Thompson IM, Tangen CM, Paradelo J, Lucia S, Miller G, Troyer D, et al. Adjuvant radiotherapy for pathologically advanced prostate cancer. JAMA 2006; 296: 2329-35.

2. Bolla M, van Poppel H, Colette L, van Cangh $\mathrm{P}$, Vekemans K, Da Pozzo L, et al. Postoperative radiotherapy after radical prostatectomy: a randomised controlled trial (EORTC trial 22911). Lancet 2005; 366: 52-8.

3. Wiegel T, Bottke D, Willich H, Piechota $H$, Souchon R, Stoeckle M, et al. Phase III results of adjuvant radiotherapy (RT) versus "wait and see« (WS) in patients with pT3 prostate cancer following radical prostatectomy (RP)(ARO 96-02/AUO AP 09/95). [Abstract]. J Clin Oncol 2005; 23(Part 1 Suppl): $381 S$.

4. Stephenson AJ, Scardino PT, Kattan MW, Pisansky TM, Slawin KM, Klein EA, et al. Predicting the outcomes of salvage radiation therapy for recurrent prostate cancer after radical prostatectomy. J Clin Oncol 2007; 25: 2035-41.

5. Jani $A B$, Sokoloff $M$, Shalhav A, Stadler W. Androgen ablation adjuvant to postprostatectomy radiotherapy: complication-adjusted number needed to treat analysis. Urology 2004; 64: 97681.

6. Zelefsky MJ, Aschkenasy E, Kelesen S, Leibel SA. Tolerance and early outcome results of postprostatectomy three-dimensional conformal radiotherapy. Int J Radiat Biol Phys 1997; 39: 327-33.

7. Cox JD, Gallagher MJ, Hammond EH, Kaplan RS, Schellhammer PF. Consensus statement on radiation therapy of prostate cancer: guidlines for prostate re-biopsy after radiation and for radiation therapy with rising prostate specific antigen levels after radical prostatectomy. American Society for Terapeutic Radiology and Oncology consensus panel. J Clin Oncol 1999; 17: 1155-63.

8. Poortmans P, Bossi A, Vandeputte K, Bosset M, Miralbell R, Maingon P, et al. Guidlines for target volume definition in post-operative radiotherapy for prostate cancer, on behalf of the EORTC Radiation Oncology Group. Radiother Oncol 2007; 84: 121-7.
9. Spiotto MT, Hancock SL, King CR. Radiotherapy after prostatectomy: improved biochemical relaps-free survival with whole pelvic compared with prostate bed only for high-risk patients. Int J Radiat Biol Phys 2007; 69: 54-61.

10. Storey MR, Pollack A, Zagars G, Smith L, Antolak J, Rosen I. Complications from radiotherapy dose escalation in prostate cancer: preliminary results of a randomized trial. Int J Radiat Biol Phys 2000; 48: $635-42$.

11. Parmar MKB, Machin D. Survival analysis. Chichester: John Wiley\&Sons; 1995.

12. Feng $\mathrm{M}$, Hanlon AL, Pisansky TM, Kuban $\mathrm{D}$, Catton C, Michalski J, et al. Predictive factors for late genitourinary and gastrointestinal toxicity in patients with prostate cancer treated with adjuvant or salvage radiotherapy. Int J Radiat Biol Phys 2007; 68: 1417-23.

13. Valicenti RK, Gomella LG, Ismail M, Mulholland SG, Petersen RO, Corn BW. Effects of higher radiation dose on biochemical control after radical prostatectomy for $\mathrm{pT} 3 \mathrm{~N} 0$ prostate cancer. Int J Radiat Biol Phys 1998; 42: 501-6.

14. Jereczek-Fossa BA, Zerini D, Vavassori A. Sooner or later? Outcome analysis of 431 prostate cancer patients treated with postoperative or salvage radiotherapy. Int J Radiat Oncol Biol Phys 2008 74: $115-25$.

15. Pearse M, Choo R, Danjoux C, Gardner S, Morton G, Szumacher E, et al. Prospective assessment of gastrointestinal and genitourinary toxicity of salvage radiotherapy for patients with prostate-specific antigen relaps or local recurrence after radical prostatectomy. Int J Radiat Biol Phys 2008; 72: 792-8.

16. Pinkawa M, Fischedick K, Asadpour B, Gagel B, Piroth MD, Eble MJ. Low-grade toxicity after conformal radiation therapy for prostate cancer - impact of bladder volume. Int J Radiat Biol Phys 2006; 64: 835-41.

17. Harsolia A, Vargas C, Yan D, Brabbins D, Lockman D, Liang J, et al. Predictors for chronic urinary toxicity after the treatment of prostate cancer with adaptive three-dimensional conformal radiotherapy: dose-volume analysis of a phase II dose-escalation study. Int J Radiat Biol Phys 2007; 69: 1100-9.

18. Portelance L, Chao KSC, Grigsby PW, Benet H, Low D. Intensity-modulated radiation therapy (IMRT) reduces small bowel, rectum and bladder dose in patients with cervical cancer receiving pelvic and para-aortic radiation. Int J Radiat Biol Phys 2001; 51: 261-6. 
19. Roeske JC, Lujan A, Rotmensch J, Waggoner SE, Yamada D, Mundt AJ. Intensity-modulated whole pelvic radiation therapy in patients with gynecologic malignancies. Int J Radiat Biol Phys 2000; 48: 1613-21.

20. Heidenreich A, Ohlmann $\mathrm{CH}$, Polyakov S. Anatomical extent of pelvic lymphadenectomy in bladder and prostate cancer. Eur Urol Suppl 2005; 4: 15-24.

21. Roach M 3rd, Marquez C, Yuo HS. Predicting the risk of lymph node involvement using the pretreatment prostatic specific antigen and Gleason score in men with clinically localized prostate cancer. Int J Radiat Biol Phys 1994; 28: 33-7.

22. Shih HA, Harisinghani M, Zietman AL, Wolfgang JA, Saksena M, Weissleder R. Mapping of nodal disease in locally advanced prostate cancer: rethinking the clinical target volume for pelvic nodal irradiation based on vascular rather than bony anathomy. Int J Radiat Biol Phys 2005; 63: $1262-9$.

23. Corn BW, Winter K, Pilepich MV. Does androgen suppression enhance the efficacy of postoperative irradiation? A secondary analysis of RTOG 85-13. Urology 1999; 54: 495-502.

24. Taussky D, Bae K, Bahary JP. Does timing of androgen deprivation influence radiation-induced toxicity? A secondary analysis of radiation therapy oncology group protocol 9413. Urology 2008; 72: 1125-9.

25. Cox JD, Stetz JA, Pajak TF. Toxicity criteria of the radiation therapy oncology group (RTOG) and the European organization for research and treatment of cancer (EORTC). Int J Radiat Biol Phys 1995; 31: 1341-6.

26. Botros M, Quevedo JF, Miller RC. Angiosarcoma of the liver after multimodality therapy for gallbladder carcinoma. Radiol Oncol 2009; 43: 126-31.

27. Velenik V, Oblak I, Anderluh F. Quality of life in patients after combined modality treatment of rectal cancer: Report of a prospective phase II study. Radiol Oncol 2008: 42: 207-14. 